\title{
NB-IoT for Satellite Communications: Physical Layer Analysis and Performance
}

\author{
Valérian Mannoni ${ }^{1}$, Vincent Berg ${ }^{1}$, Sonia Cazalens ${ }^{2}$, Patrice Raveneau ${ }^{2}$ \\ ${ }^{1}$ CEA-Leti, Université Grenoble Alpes, F-38000 Grenoble, France \\ 2 CNES, 18 avenue Edouard Belin, 31400 Toulouse, France \\ $\{$ valerian.mannoni, vincent.berg\}@cea.fr, \{sonia.cazalens, patrice.raveneau\}@cnes.fr
}

\begin{abstract}
Terrestrial Internet of Things (IoT) communication systems cannot provide worldwide coverage as effectively as satellite systems. Narrow Band IoT (NB-IoT) is currently one of the major terrestrial IoT technology. This article provides an evaluation of NB-IoT physical layer performance in the context of two satellite scenarios: Geostationary (GEO) and Low Earth Orbit (LEO). GEO scenarios suffer from large distances between transmitter and receiver, while LEO systems exhibit very large levels of Doppler. New algorithms are proposed to reliably adapt NB-IoT in these contexts and performance in terms of maximum throughput is evaluated and compared. Finally, NB-IoT main limitations in satellite communication links are identified.
\end{abstract}

Keywords-NB-IoT, Satellite Communications, LEO, GEO, Physical Layer, Doppler

\section{INTRODUCTION}

The Internet of Things (IoT) revolution offers huge potential benefits in terms of improved efficiency, sustainability and safety for the industry and society. The variety of applications and solutions designed for individuals, businesses and industries is spurring the rapid expansion of the IoT market. The IoT is playing a major role across a variety of vertical sectors, generating cost savings and new revenue streams. Analysts predict strong growth in the number of IoT connections from around 12 billion today to 31 billion in 2025. Among the possible IoT connectivity technologies, new approaches often referred as Low Power Wide Area (LPWA) networking have emerged. These technologies give a low power connectivity alternative to current generations of cellular systems, while covering large areas. The main LPWA communication systems are: Narrow Band IoT (NB-IoT) and Semtech Long Range (LoRA). In H1 2020, $44 \%$ of IoT LPWA connections were performed with LoRA and $46 \%$ with NBIoT [1]. While LoRa is a proprietary solution, NB-IoT is an alternative proposed by the 3GPP standard body to enable a wide range of cellular devices and services with a long lasting battery life compared to the classical cellular radio. NB-IoT uses a subset of the LTE standard but limits the bandwidth to $200 \mathrm{kHz}$. It uses Orthogonal Frequency-Division Multiplexing Access (OFDMA) modulation for downlink (DL) and SingleCarrier Frequency Division Multiple Access (SC-FDMA) for uplink (UL) communications. IoT applications which require more frequent communications will be better served by NBIoT than LoRA, as no duty cycle limitations are required on the licensed spectrum.

NB-IoT is therefore designed for a terrestrial deployment. However today, some applications do not have access to a global, reliable IoT connectivity service and are unable to reach remote assets due to erratic or insufficient coverage. Satellite communications will then provide a complementary connectivity for the IoT. Whether fixed or on the move, across land and ocean, satellite communications will simplify the way businesses access and use data from assets, no matter where they are deployed, in a simple and affordable way. Existing satellite systems (Iridium, Globalstar, Orbcomm, etc.) have already proven their efficiency for standard voice/data applications but remain too expensive for most IoT applications. New generation of satellite links dedicated to the IoT are therefore being studied and should be developed in the coming years and the main challenge for the satellite IoT community is to choose the best technology. This is why we propose to analyze the compatibility of the NB-IoT cellular standard with bidirectional satellite links in Geostationary Orbit (GEO) and in Low Earth Orbit (LEO). Also note that a 3GPP study item has started in 2021 to address satellite NB-IoT and to discuss its adaptations.

In this context, very little work has been so far documented in the literature. A preliminary link budget study has recently been performed for a LEO satellite constellation in [2]. However, the contributions mainly discussed the Doppler effect for LEO scenarios by analyzing it and by proposing different approaches to limit its impact in [3] [4]. In this article we propose a complete receiver architecture to communicate with GEO and LEO satellites. The performance results are compared based on link budgets derived from a study of the scenarios. This paper takes the approach to analyze the 3GPP standard of NB-IoT and provide as much contained modifications as possible with regards to standardized NB-IoT.

After a description of the NB-IoT standard in section II-A, the satellite use cases/scenarios are described in details in section II-B in order to deduce link budgets and propagation models in section II-C. The link level performance of the NBIoT physical layer is then derived and presented in section III-D. Finally, section IV concludes the document with a synthesis of the evaluation.

\section{SySTEM MODEL}

\section{A. NB-IoT standard}

NB-IoT was conceived as an evolution of the LTE system. This section presents NB-IoT Release 15 and in particular its physical layer with the over-the-air frame structure and traffic exchanges with the eNodeB: UL and DL physical channels. On the physical layer, NB-IoT adopts the same numerology as legacy LTE along with OFDMA and SC-FDMA waveforms in 


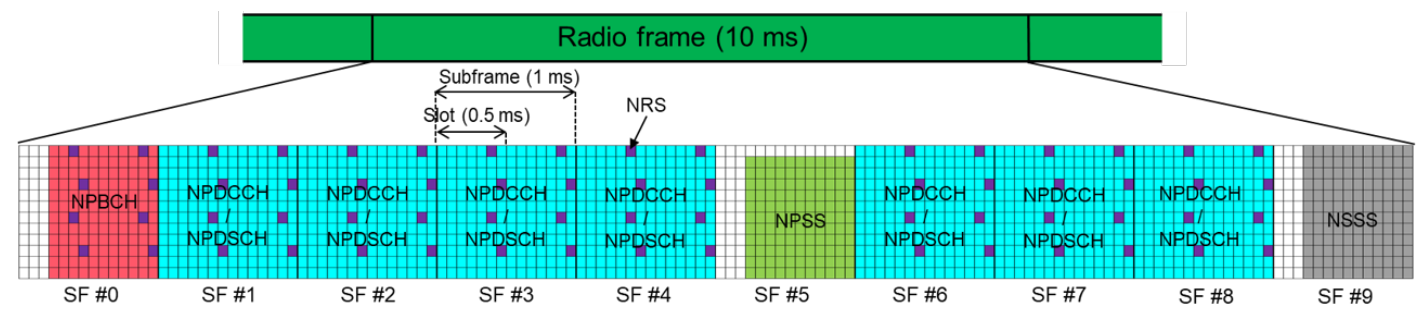

Fig. 1. Downlink frame structure for $\Delta f=15 \mathrm{kHz}$.

DL and UL, respectively [5] [6]. The frame structure, depicted in Fig. 1 for DL, is function of the subcarrier spacing $\Delta f$. NBIoT supports two modes of $\Delta f$ i.e. $15 \mathrm{kHz}$ for $\mathrm{DL}$ and $\mathrm{UL}$ and $3.75 \mathrm{kHz}$ for UL only. A radio frame lasts $10 \mathrm{~ms}$ and consists of 20 slots of length $0.5 \mathrm{~ms}$ when $\Delta f=15 \mathrm{kHz}$. For $\Delta f=3.75 \mathrm{kHz}$, a radio frame corresponds to 5 slots of $2 \mathrm{~ms}$. A subframe is defined as two consecutive slots. Multiplexing relies on half-duplex frequency-division duplexing. In DL, the signal bandwidth of $180 \mathrm{kHz}$ is divided into 12 subcarriers of $\Delta f=15 \mathrm{kHz}$. UL is more flexible with a number of active subcarriers that can be: $1,3,6$ and 12 for $\Delta f=15 \mathrm{kHz}$ with a resulting bandwidth spanning from $15 \mathrm{kHz}$ to $180 \mathrm{kHz}$. In UL when $\Delta f=3.75 \mathrm{kHz}$, NB-IoT uses a single carrier waveform with a resulting bandwidth of $3.75 \mathrm{kHz}$.

1) Downlink NB-IoT physical layer: Three different physical channels can be transmitted in DL [7] [6], the Narrowband Physical Downlink Shared Channel (NPDSCH) for the data, the Narrowband Physical Broadcast Channel (NPBCH) for the broadcast control (which carries the narrowband master information block MIB-NB) and the Narrowband Physical Downlink Control Channel (NPDCCH) for the control. Three synchronization and channel estimation signals are available: Narrowband Primary Synchronization Signals (NPSS), Narrowband Secondary Synchronization Signals (NSSS) and Narrowband Reference Signal (NRS). The scheduling of these physical channels and synchronization signals is shown through the resource grid given in Fig. 1. A slot consists of seven consecutive OFDM symbols. To transmit a transport block, a CRC is added and the information bits are encoded by a convolutional code. The codeword is then rate matched to reach the targeted data rate, interleaved and scrambled. The output is then modulated using QPSK. Finally, the resulting complex symbols supply the CP-OFDM modulator. More details can be found in [8].

2) Uplink NB-IoT physical layer: Two physical channels are used in UL [7] [6]: the Narrowband Physical Uplink Shared Channel (NPUSCH) for data/control and the Narrowband Physical Random Access Channel (NPRACH). The UL signal is Narrowband demodulation reference signal. The transmission scheme is based on the SC-FDMA waveform which consists in a DFT precoding before OFDM modulation reducing the PAPR of the waveform and thus increasing the battery life of the device [9]. NPUSCH is used to transmit UL transport block and then performs the following functionalities: CRC insertion, Turbo coding, rate matching, scrambling and mapping. The resulting symbols are then DFT-spread and OFDM modulated.

NPRACH is the physical channel used to transmit the random access preamble during the random access procedure that enables a user equipment (UE) to establish a connection with the network (switch from Idle to Connected mode). Single tone signal frequency hopping $(\mathrm{FH})$ algorithms have been designed for NPRACH to obtain time and frequency diversity gain. The instantaneous signal bandwidth is $3.75 \mathrm{kHz}$. A preamble consists of 4 symbols grouped as a basic unit, which is transmitted via 4 different sub-carriers using a $\mathrm{FH}$ mechanism [6]. Note that collisions cannot be prevented via the $\mathrm{FH}$, because all sub-carriers in the $\mathrm{FH}$ scheme are a priori determined by the parameter $P H Y_{C E L L}^{I D}$.

\section{B. Satellite Scenarios}

Satellite propagation scenarios significantly differ from their terrestrial counterparts as the eNodeB is much further apart from the UE and is constantly moving when non Geostationary satellites are considered. Two scenarios of IoT satellites have been considered: GEO and LEO satellites. The LEO scenario considered satellites at an altitude of $770 \mathrm{~km}$ with an inclination of $84.6^{\circ}$. The complete surface of the Earth requires three GEO satellites to achieve coverage, while 66 satellites would be required to constantly provide coverage for the LEO scenario.

For both satellite scenarios, multi-spot antenna patterns are considered (Fig. 2). Mechanical analysis of both scenarios are used to derive the distances and the radial speed and radial speed variations between the satellite eNodeB and NB-IoT UE transceivers on the surface of the Earth.

For the GEO scenario, the satellite is located at a fixed longitude in an Earth Centered, Earth Fixed (ECEF) coordinate. For any given UE located on the surface of the Earth in the area of coverage of the satellite, distance between satellite and UE is therefore fixed, while radial speed is constant and null. Therefore, the Doppler value is null. This scenario considers a 139-spot antenna pattern (Fig. 2). Each spot is distant from each other in the $(\mathrm{B}, \mathrm{A})$ angular/polar plan by $1.56^{\circ}$.
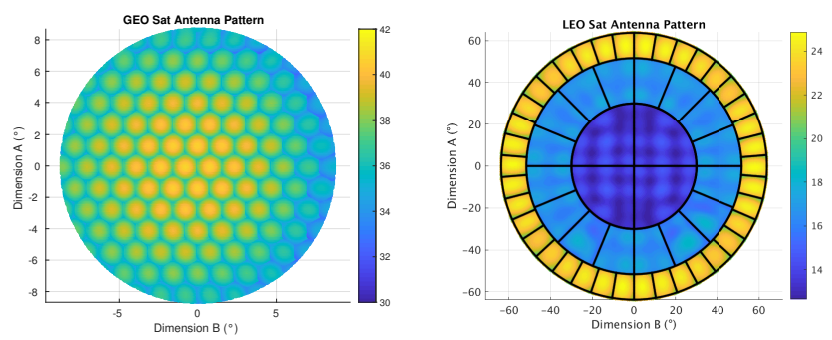

Fig. 2. Spot antenna pattern for GEO (left) and LEO (right) scenarios. 
TABLE I. PHYSICAL PARAMETERS FOR THE GEO SCENARIO

\begin{tabular}{|c|c|c|c|c|c|c|}
\hline Spot Angle & $\mathbf{0}^{\circ}$ & $\mathbf{1 . 5 6}^{\circ}$ & $\mathbf{3 . 1 2}^{\circ}$ & $\mathbf{4 . 6 8}^{\circ}$ & $\mathbf{6 . 2 4}^{\circ}$ & $\mathbf{7 . 8 0}^{\circ}$ \\
\hline Distances (km) & 35786 & 35890 & 36211 & 36780 & 37678 & 39186 \\
\hline
\end{tabular}

This geometrical property is used to evaluate the distance distribution intervals between the satellite and a point on the surface of the earth as a function of the distance (in ${ }^{\circ}$ ) from the center of the antenna footprint on Earth. We proposed to separate the distance distributions into 5 intervals as a function of the angle from the antenna pointing towards the center of the Earth. Distance levels are summarized in Table I.

For the LEO scenario, a 58-spot antenna is considered. It consists of 3 rings of respectively 4,16 and 38 sectors. Earth satellite orbits have been simulated by solving Kepler's equation using Newton's method. The distributions of link distances, radial speed and radial speed variations are evaluated for a uniform distribution of UE located on the surface of the Earth. These metrics are then converted into Doppler and Doppler variations (Table II).

\section{Link Budget}

Link budgets for the scenarios described in section II-B are here derived. UL (from the Earth to the Satellite) and DL (from the Satellite to the IoT node) are considered separately. While Satellites are assumed regenerative, results also apply to transparent satellites when data link from Earth Station to satellite is not limiting transmission. For UL, power received at the satellite antenna is given by:

$$
\left[P_{i n S a t}\right]_{d B m}=\left[P_{U E}\right]_{d B m}+\left[G_{U E}\right]_{d B}-[L]_{d B},
$$

Where $\left[P_{U E}\right]_{d B m}$ is the transmit power of the UE in $\mathrm{dBm}$, $\left[G_{U E}\right]_{d B}$ the antenna gain of the UE and $[L]_{d B}$ corresponds to the propagation losses in $\mathrm{dB}$. It includes free space loss but also additional losses such as atmospheric and polarisation losses. Free space loss is function of the signal carrier frequency. Then, carrier-to-noise power ratio $\left[C_{R x S a t} / N\right]_{d B}$ is given by:

$$
\begin{aligned}
{\left[\frac{C_{R x S a t}}{N}\right]_{d B} } & =\left[P_{\text {inSat }}\right]_{d B m}+\left[\frac{G_{s}}{T_{s}}\right]_{d B / K} \\
& -[k]_{d B J / K}-\left[B_{U L}\right]_{d B H z}
\end{aligned}
$$

Where $\left[G_{s} / T_{s}\right]_{d B / K}$ is a specification input of the satellite receiver, $[k]_{d B J / K}$, the Boltzmann constant expressed in $\mathrm{dB}$ and equal to $-228.6 \mathrm{~dB} . \mathrm{J} / \mathrm{K},\left[B_{U L}\right]_{d B H z}$ the bandwidth of the UL signal in $\mathrm{dB}$. Similarly, for DL operation, the power received at the NB-IoT node, $\left[P_{i n U E}\right]_{d B m}$, is given by:

$$
\left[P_{i n U E}\right]_{d B m}=\left[P_{E I R P-S A T}\right]_{d B m}-[L]_{d B}
$$

where $\left[P_{E I R P-S A T}\right]_{d B m}$ is the equivalent isotropic radiated power at the satellite and $[L]_{d B}$ corresponds to the propagation

\begin{tabular}{|c|c|c|}
\hline Parameter & Value & Comment \\
\hline$\left[P_{U E}\right]_{d B m}$ & $23 \mathrm{dBm}$ & RMS Transmit power in $\mathrm{dBm}$ \\
\hline$\left[G_{U E}\right]_{d B}$ & $-1.5 \mathrm{~dB}$ & UE transmitter and receiver antenna gain \\
\hline$f_{U L}$ & $1643 \mathrm{MHz}$ & Uplink Frequency \\
\hline$f_{D L}$ & $1545 \mathrm{MHz}$ & Downlink Frequency \\
\hline$\left[P_{E I R P}-S A T\right]_{d B m}$ & scenario & EIRP of Satellite in $\mathrm{dBm}$ \\
\hline$\left[G_{s} / T_{s}\right]_{d B / K}$ & scenario & G/T of the satellite \\
\hline$\left[L_{A t m o s}\right]_{d B}$ & $0.5 \mathrm{~dB}$ & Additional atmospheric losses \\
\hline$\left[L_{\text {Polar }}\right]_{d B}$ & $3 \mathrm{~dB}$ & Polarization losses \\
\hline$\left[B_{U L}\right]_{d B H z}$ & $\begin{array}{c}3.75 \mathrm{kHz} \\
15 \mathrm{kHz} \\
45 \mathrm{kHz} \\
90 \mathrm{kHz} \\
180 \mathrm{kHz}\end{array}$ & $\begin{array}{c}\text { UL Bandwidth } \\
\text { (multiple options are considered) }\end{array}$ \\
\hline$\left[B_{D L}\right]_{d B H z}$ & $180 \mathrm{kHz}$ & DL Bandwidth \\
\hline$\left[N F_{R x U E}\right]_{d B}$ & $8 \mathrm{~dB}$ & Noise Figure of the UE receiver \\
\hline
\end{tabular}

TABLE II. Physical PARAMETERS FOR THE LEO SCENARIO

\begin{tabular}{|l|l|l|l|}
\hline Spot Location & Distances $(\mathbf{k m})$ & Doppler $(\mathbf{k H z})$ & $\begin{array}{l}\text { Doppler } \\
\text { Variations }(\mathbf{H z} / \mathbf{s})\end{array}$ \\
\hline Sat Coverage & $780-2617 \mathrm{~km}$ & $-36 \mathrm{kHz}-36 \mathrm{kHz}$ & $5-345 \mathrm{~Hz} / \mathrm{s}$ \\
\hline Center ring & $780-904 \mathrm{~km}$ & $-20 \mathrm{kHz}-20 \mathrm{kHz}$ & $225-345 \mathrm{~Hz} / \mathrm{s}$ \\
\hline Ring 1 & $905-1305 \mathrm{~km}$ & $-31-31 \mathrm{kHz}$ & $71-300 \mathrm{~Hz} / \mathrm{s}$ \\
\hline Ring 2 & $1306-2617 \mathrm{~km}$ & $-36-36 \mathrm{kHz}$ & $5-204 \mathrm{~Hz} / \mathrm{s}$ \\
\hline
\end{tabular}

TABLE III. TRANSMISSION PARAMETERS FOR THE LINK BUDGET.

losses in $\mathrm{dB}$ for $\mathrm{DL}$ frequency. Finally, the carrier-to-noise power ratio $\left[C_{R x U E} / N\right]$ at the NB-IoT receiver is given by:

$$
\begin{aligned}
{\left[\frac{C_{R x U E}}{N}\right]_{d B} } & =\left[P_{i n U E}\right]_{d B m}+\left[G_{U E}\right]_{d B}+174 \\
& -\left[N F_{R x U E}\right]_{d B}-\left[B_{D L}\right]_{d B H z}
\end{aligned}
$$

Where $\left[G_{U E}\right]_{d B}$ is the gain of the NB-IoT antenna receiver, $\left[N F_{R x U E}\right]_{d B}$, the Noise Figure of the receiver, $\left[B_{D L}\right]_{d B H z}$ the bandwidth of the DL signal.

No margin has been included in this link budget. This should be considered in the interpretation of the data. RMS transmit power of the NB-IoT UE is set to $200 \mathrm{~mW}$ or $23 \mathrm{dBm}$ in this section. Analysis may consider $200 \mathrm{~mW}, 500 \mathrm{~mW}$ and $1000 \mathrm{~mW}$ scenarios, in this case, UL link budgets should be increased by respectively $0 \mathrm{~dB}, 4 \mathrm{~dB}$ and $7 \mathrm{~dB}$. $\left[P_{E I R P-S A T}\right]_{\mathrm{dBm}}$ is function of the scenario and the ring where signal is scattered. Table III gives the hypothesis that have been considered. Satellite parameters, EIRP and $\left[G_{s} / T_{s}\right]_{d B / K}$, are given for each scenario in Table IV.

TABLE IV. SATEllite EIRP For GEO AND LEO SCENARIOS.

\begin{tabular}{|c|c|c|c|}
\hline Scenario & Spot Location & $\begin{array}{c}\text { EIRP in the } \\
\text { spot }(\mathrm{dBm})\end{array}$ & $\begin{array}{c}\mathbf{E}\left[G_{s} / T_{s}\right]_{d B / K} \\
(\mathrm{~dB} / \mathrm{K})\end{array}$ \\
\hline \multirow{2}{*}{ GEO } & Center & 82.3 & 9.4 \\
& Edge & 79.8 & 6.9 \\
\hline \multirow{2}{*}{ LEO } & Ring 1 & 50.5 & 20.5 \\
& Ring 2 & 53.9 & 23.9 \\
& Ring 3 & 58.9 & 28.9 \\
\hline
\end{tabular}

UL (resp. DL) communication link budget results for the GEO and LEO scenarios are given in Fig. 3 (resp. Fig. 4). For UL communication, the link budget is not only dependent on the position on the satellite spot, but also on the UL signal bandwidth. For the GEO scenario, gain is varying by approximately $2.5 \mathrm{~dB}$ within a spot (Fig. 2). $\left[C_{R x S a t} / N\right]_{d B}$ varies for this case from $-17.7 \mathrm{~dB}$, when $180 \mathrm{kHz}$ bandwidth is considered in the edge of the spot to $2.4 \mathrm{~dB}$ in the center of a spot when $3.75 \mathrm{kHz}$ bandwidth is considered. For LEO, $\left[C_{R x \text { Sat }} / N\right]_{d B}$ varies from $-8.8 \mathrm{~dB}$ to $13.8 \mathrm{~dB}$ depending on the bandwidth. In this scenario, the gain of the satellite antenna is larger in the spots located in the outer rings than in the spots in the central ring. This supplementary gain compensates for the larger distances when the signal is transmitted through the outer rings. 


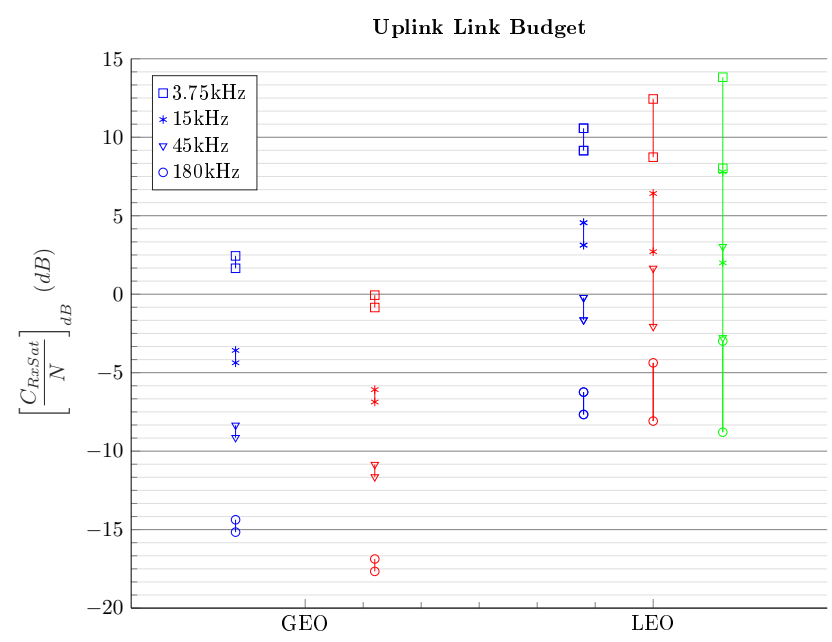

Fig. 3. Uplink link budget for GEO and LEO scenarios. Colors show the ring number of the Satellite antenna

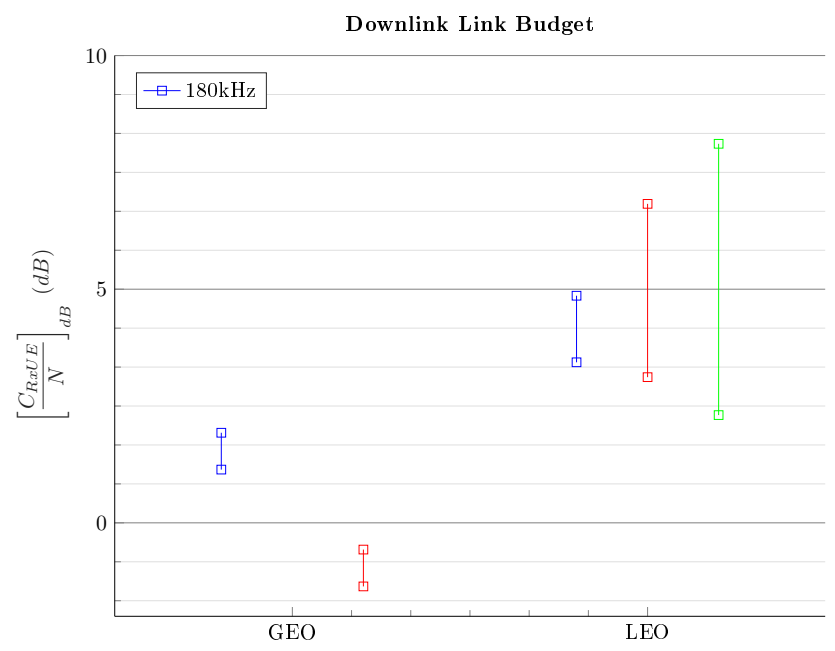

Fig. 4. Downlink link budget for GEO and LEO scenario. Carrier-to-noise ratio at the NB-IoT Terminal

\section{PERFORMANCE EVALUATION}

This section provides an overview of the NB-IoT receiver architecture and the associated approach to be able to communicate via a LEO satellite. Indeed, unlike the GEO satellite, the high level of Doppler encountered during communications with a LEO satellite requires us to implement a specific strategy. The performance for the different physical channels (NPBCH/NPDCCH/NPDSCH in DL, NPUSCH/NPRACH in $\mathrm{UL})$ will be then derived.

\section{A. NB-IoT receiver strategy for satellite communications}

In this subsection we describe the procedure required to communicate with NB-IoT via a LEO satellite.

A UE starts with a cell search procedure to acquire time and frequency synchronization with a cell and detects the narrowband physical layer Cell ID with the synchronization signals (NPSS and NSSS). Based on this synchronization, a UE has to decode the NBPCH channel in order to obtain the some basic system information (MIB-NB). The NPBCH transmission time interval is $640 \mathrm{~ms}$ and is transmitted in 64 parts, each part is mapped to the first subframe of a radio frame (Fig. 1)

eNodeB uses then Downlink Control Information on NPD$\mathrm{CCH}$ to specify the scheduling information for a DL/UL transmission in NB-IoT. Thus, the UE has to demodulate NPDCCH channel to find out whether it has data addressed to it in subsequent NPDSCH channels or whether it has radio resource to transmit an NPUSCH channel.

It is important to note that $3 \mathrm{GPP}$ recommands that the NB-IoT UE modulated carrier frequency shall be accurate to within the limits of +/- $0.1 \mathrm{ppm}$ (i.e. $+/-160 \mathrm{~Hz}$ at $1.6 \mathrm{GHz}$ ) compared to the carrier frequency received from the eNodeB [10]. This implies then that the frequency offset should be accurately estimated on DL (during the synchronization step) and compensated by the IoT device when transmitted on UL. This step is essential to minimize the frequency offset on UL so that it can be efficiently estimated and corrected. Indeed, the signaling on the UL frame only allows us to estimate a maximum frequency offset of $+/-1 \mathrm{kHz}$, whereas the scenario analysis showed a frequency offset up to $+/-36 \mathrm{kHz}$. This monitoring of the frequency offset on the DL channel must therefore be performed regularly at least every $300 \mathrm{~ms}$ because of the fast variations of the Doppler level.

\section{B. Downlink physical layer decoding}

One of the key requirements for optimum demodulation of OFDM signals of the NPBCH/NPDCCH/NPDSCH channels is that there is accurate frequency and timing synchronization. Timing synchronization is the first step to be accomplished during initial access. It has been chosen to rely on a correlation in the time domain with NPSS for this synchronization. This approach is the most robust (compared to autocorrelation on the CP) but it is necessary to properly combine coherent and non-coherent accumulations in order to minimize the impact of frequency offset. The synchronization process performs a correlation between the received signal and the NPSS signal. This correlation takes place in the time domain and is computed OFDM symbol by OFDM symbol to ensure a robust Carrier Frequency Offset (CFO) estimation. The channel considered for satellite communications is the Gaussian channel. Thus, the received signal is,

$$
r(k)=c_{k} e^{j\left(2 \pi f_{d} k T_{s}+\theta\right)}+n(k), k=0, \ldots, N_{f f t}+N_{G I}
$$

with $c_{k}$ the transmitted symbol (in the time domain), $T_{s}$ the sampling period, $f_{d}$ the frequency offset (assumed constant for the frame duration), $\theta$ the carrier phase and $n(k)$ the additive noise. The time correlation with the transmitted sequence can then be written as

$$
\begin{array}{r}
C_{n_{s}}^{n_{F R}}(n)=\sum_{k=0}^{N_{c}} r\left(k+n+n_{s}\left(N_{c}\right)+n_{F R} K_{F R}\right) \\
s_{N P S S}^{*}\left(k+n_{s}\left(N_{c}\right)\right), n_{s}=2, \ldots, 13, n_{F R}=0, \ldots, 63 .
\end{array}
$$

$K_{F R}$ represents the number of samples in a radio frame, $N_{c}=N_{f f t}+N_{C P}$ with $N_{f f t}$ the FFT size and $N_{C P}$ the prefix cyclic size. We then perform a non-coherent accumulation of these correlations to detect the presence of signal and the starting position. The performance of this detection 
approach is directly dependent on the number of points used in the aggregated correlations. Thus, we correlated the received signal with the NPSS on $N_{F R}=64$ radio frames $(\mathrm{NPBCH}$ duration) to address SNR levels that are low enough not to be the limiting factor in the reception chain,

$$
R(n)=\sum_{n_{F R}=0}^{N_{F R}-1} \sum_{n_{s}=2}^{13}\left|C_{n_{s}}^{n_{F R}}(n)\right|^{2} .
$$

The CFO estimation problem can be solved in two independent steps with a first estimation of the fractional part of $f_{d} / \Delta f$ which will then be completed by estimating the integer part. The integer part of $f_{d} / \Delta f$ will generate cyclic shift of the modulated data and lead to detection error at the receiver while the fractional part will cause inter-carrier interference (ICI) and SNR degradation. Then, suitable methods should be used to correctly estimate and compensate CFO. The fractional part of $f_{d} / \Delta f$ is easily detectable in the time domain by exploiting the angle of the correlation processed during the time synchronization at the detection point, i.e. the maximal autocorrelation point. The correlation with the transmitted sequence can then be written as $C(k)=e^{j\left(2 \pi f_{d} k T_{s}+\theta\right)}+n(k) c_{k}^{*}$. The proposed estimator exploits then the sample correlations,

$$
A(m)=1 /\left(L_{0}-m\right) \sum_{k=m}^{L_{0}-1} C(k) C^{*}(k-m), 1 \leq m \leq N
$$

where $\mathrm{N}$ is a design parameter not greater than $L_{0} / 2$. Thus, from the equation (8) we can use the frequency offset estimator introduced by Mengali in [11] which presents good performance for burst-mode transmissions (as NPSS).

The integer part can be detected in a post-FFT processing using NPBCH or using the CRC check after NPBCH decoding. Thus, a simple way to estimate the integer frequency error is to iteratively add $k \Delta f(\mathrm{k}=0,1,-1,2,-2,3,-3)$ and test the $\mathrm{CRC}$ to check if the packet has been successfully decoded (CRC fulfilment is then the criteria for stopping the iterative process). As soon as the CRC is valid, the frequency offset is considered to be found. After the synchronization process, the receiver is similar to the terrestrial one.

\section{Uplink physical layer decoding}

As for DL, NPUSCH time synchronization will be achieved by correlation in the time domain. The major difference is that on UL there is no dedicated synchronization signal, so we have exploited the reference symbols (DRS) which are rather dedicated to channel estimation and power control. The absence of a synchronization signal in UL is mainly due to the fact that there is no major synchronization issue in UL when terrestrial NB-IoT is considered. Since the eNodeB has allocated resources and has the "Timing Advance" information, it knows when it would receive the TB transmitted by the UE. In the satellite case, a time synchronization to find the frame start is however required but with a very small search space. In the context of satellite communications, since the propagation times are much longer, time synchronization must be performed with a much larger search space (on several slots). Thus, to obtain good performance on time synchronization, especially for low SNR levels, it is required that the reference symbols included in each slot are different to avoid the slot ambiguity. The standard offers this possibility by enabling the "Sequence-group hopping" [6]. It gives the number of reference signal sequences available for each resource unit size in terms of number of subcarriers. For example, 30 different reference symbols are possible when the subcarriers number is 12. Thus, for UL synchronization, time domain correlation is performed with each different reference symbols.

To estimate the frequency offset in UL we will have to estimate the evolution of the phase between two reference symbols. To do this, we will use the correlations calculated during the time synchronization. Indeed, the evolution of the phase of the correlation between two successive reference symbols directly gives the frequency offset, as:

$$
\begin{array}{r}
\tilde{\phi}_{j}=\arg \left(C_{j+1}\left(N_{\text {synchro }}\right)\right)-\arg \left(C_{j}\left(N_{\text {synchro }}\right)\right), \\
j=0, \ldots, N_{\text {slot }}-2 .
\end{array}
$$

The estimation accuracy is then improved by averaging over all the reference symbols included in the UL frame. The maximum CFO that can be estimated is given by the delay between two reference symbols. The phase of the correlation must not be rotated by more than $2 \pi$ between both symbols, otherwise there is an ambiguity of a factor of $2 \pi$ on the frequency offset estimate. Thus, we can only estimate a CFO of $+/-1 \mathrm{kHz}$ in UL. It is therefore important to have an accurate estimate of the DL carrier frequency so that you never have more than $1 \mathrm{kHz}$ of frequency offset in UL (including the Doppler evolution). The rest of the processing in reception of the NPUSCH channel are equivalent to the terrestrial one and does not require additional explanation.

Another part of the receiver in UL is the NPRACH detector. The objective of the NPRACH receiver is to detect the Random Access Preamble (RAP) transmitted by the UE when it tries to connect to the network. This preamble depends on its frequency location and its associated frequency hopping mechanism. For the NPRACH channel, the carrier spacing is equal to $3.75 \mathrm{kHz}$ allowing up to 48 sub-carriers in the $180 \mathrm{kHz}$ band. This band is then divided into 4 groups which means that we have 12 possible preambles per group. With the reduced carrier spacing at $3.75 \mathrm{kHz}$, the main challenge is to detect the preamble by being robust to large CFO levels. We then correlated the received signal symbol by symbol with the symbols of the possible preambles Pre,

$$
\begin{array}{r}
C_{j, p, r}^{P r e}(n)=\sum_{k=0}^{N_{f f t}-1} r\left(k+n+(j+6 p+24 r) N_{f f t}\right) \\
s_{N P R A C H, j, p}^{P r e^{*}}(k), j=0, \ldots, 5, \\
p=0, \ldots, 3, r=0, \ldots N_{r e p}-1, \operatorname{Pre}=0, \ldots, 11,
\end{array}
$$

with $N_{\text {rep }}$ the repetition rate. Then we accumulated them in a non-coherent way to support the CFO and to deduce the preamble, as

$$
\tilde{\text { Pre }}=\underset{\text { Pre }, n}{\arg \max }\left(\sum_{r=0}^{N_{\text {rep }-1}} \sum_{p=0}^{3} \sum_{j=0}^{5}\left|C_{j, p, r}^{\text {Pre }}(n)\right|^{2}\right)
$$

Thus, with this approach we can detect the preamble with a frequency offset inferior to $1 \mathrm{kHz}$. 


\section{Physical layer performance}

This section provides the simulation results for the scenarios described in section II-B. The objective is to estimate the maximum data rates for the data channels (NPDSCH, NPUSCH) and the PHY configurations for the NPDCCH and NPRACH channels. The performance will be mainly illustrated in terms of Packet Error Rate (PER) as a function of the SNR.

Fig. 5 shows the NPBCH performance for a frequency offset of $\Delta f / 2=7.5 \mathrm{kHz}$ which represents the worst case. $\mathrm{NPBCH}$ channel has to be robust because it must be decoded in order to collect information such as MIB-NB from the network. This is confirmed by the performance shown in Fig. 5 with a working point below $-16 \mathrm{~dB}$ which is inferior to the SNRs given in the link budget section. Thus, the NPBCH channel can be decoded regardless of the scenario/satellite.

The performance of the physical control channel NPDCCH for different repetition rates (from 1 to 32) with a frequency offset of $7.5 \mathrm{kHz}$ is given in Fig. 5. Thus, based on the link budgets derived in section II-C, we can identify PHY configuration with the minimum repetition rate required for the NPDCCH channel to be correctly decoded for the LEO and GEO satellite scenarios. The minimum repetition rates are then $N_{\text {rep }} \geq 1$ for the LEO and $N_{r e p} \geq 2$ for the GEO scenarios.

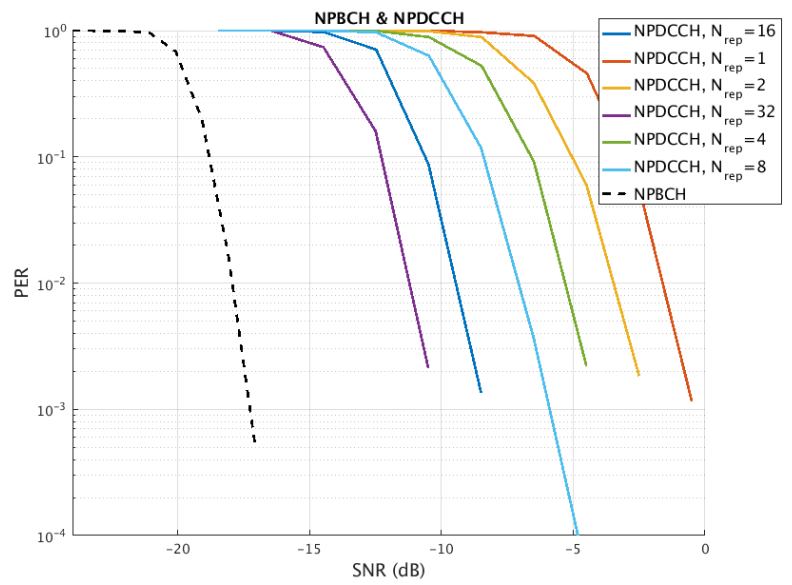

Fig. 5. NPBCH / NPDCCH performance in terms of PER vs SNR

Fig. 6 gives NPDSCH performance for different PHY configurations chosen to maximise the throughput for both scenarios (LEO and GEO). The performance is then illustrated for one transmission and with the Hybrid Automatic Repeat reQuest (HARQ) process (up to 3 transmissions). By crossing the results with the link budgets, we can see that for the LEO scenario it is possible to communicate up to $136 \mathrm{~kb} / \mathrm{s}$ (resp. 32 $\mathrm{kb} / \mathrm{s}$ ) with HARQ (resp. 1 transmission). For the GEO scenario, we can achieve a throughput of $100 \mathrm{~kb} / \mathrm{s}($ resp. $32 \mathrm{~kb} / \mathrm{s}$ ) with HARQ (resp. 1 transmission).

For UL, Fig. 7 shows the UL NPUSCH performance with HARQ process for the configurations given in Table $\mathrm{V}$ and chosen to maximize the throughput. Thus, for LEO satellite we can communicate on the UL with a data rate of $8.2 \mathrm{~kb} / \mathrm{s}$ (resp. $25.6 \mathrm{~kb} / \mathrm{s}$ and $54.7 \mathrm{~kb} / \mathrm{s}$ ) with HARQ for a transmission power of $23 \mathrm{dBm}$ (resp. $27 \mathrm{dBm}$ and $30 \mathrm{dBm}$ ). For GEO satellite, a throughput of $80 \mathrm{~b} / \mathrm{s}$ (resp. $1.28 \mathrm{~kb} / \mathrm{s}$ and $3.4 \mathrm{~kb} / \mathrm{s}$ ) can be

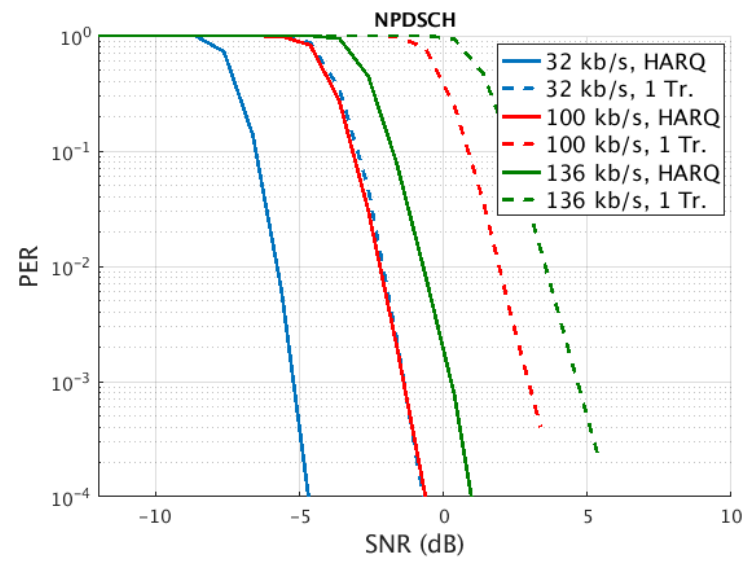

Fig. 6. NPDSCH performance.

reached with HARQ and a transmission power of $23 \mathrm{dBm}$ (resp. $27 \mathrm{dBm}$ and $30 \mathrm{dBm}$ ). As we could have anticipated, the limiting link is UL and not DL. Note that the effective throughput with the HARQ depends on the SNR. For low SNR levels, three transmissions are required while for high SNR levels only one transmission is required. We can also remark that the scenario with the GEO satellite suffers from the distance between the users and the satellite. However, it is possible under some conditions to have higher throughput if $\Delta f=3.75 \mathrm{kHz}$ is used. For example, a throughput of $320 \mathrm{~b} / \mathrm{s}$ at $23 \mathrm{dBm}$ for GEO satellite could be considered in this latter case.

TABLE V. RADIO CONFIGURATIONS FOR UL SIMULATIONS ( $\Delta f=15$ $\mathrm{KHz})$.

\begin{tabular}{|c|c|c|c|c|c|}
\hline MCS & $\begin{array}{c}\text { Nb of sub- } \\
\text { carriers }\end{array}$ & $\begin{array}{c}\text { Nb of } \\
\text { resource unit }\end{array}$ & $\begin{array}{c}\text { repetition } \\
\text { rate }\end{array}$ & $\begin{array}{c}\text { Transport } \\
\text { block size }\end{array}$ & Data rate \\
\hline 4 & 3 & 5 & 2 & $328 \mathrm{bits}$ & $8.2 \mathrm{~kb} / \mathrm{s}$ \\
\hline 3 & 12 & 5 & 2 & $256 \mathrm{bits}$ & $25.6 \mathrm{~kb} / \mathrm{s}$ \\
\hline 3 & 12 & 6 & 1 & $328 \mathrm{bits}$ & $54.7 \mathrm{~kb} / \mathrm{s}$ \\
\hline 1 & 1 & 8 & 64 & $328 \mathrm{bits}$ & $80 \mathrm{~b} / \mathrm{s}$ \\
\hline 1 & 1 & 8 & 4 & $328 \mathrm{bits}$ & $1.28 \mathrm{~kb} / \mathrm{s}$ \\
\hline 3 & 3 & 6 & 4 & $328 \mathrm{bits}$ & $3.4 \mathrm{~kb} / \mathrm{s}$ \\
\hline
\end{tabular}

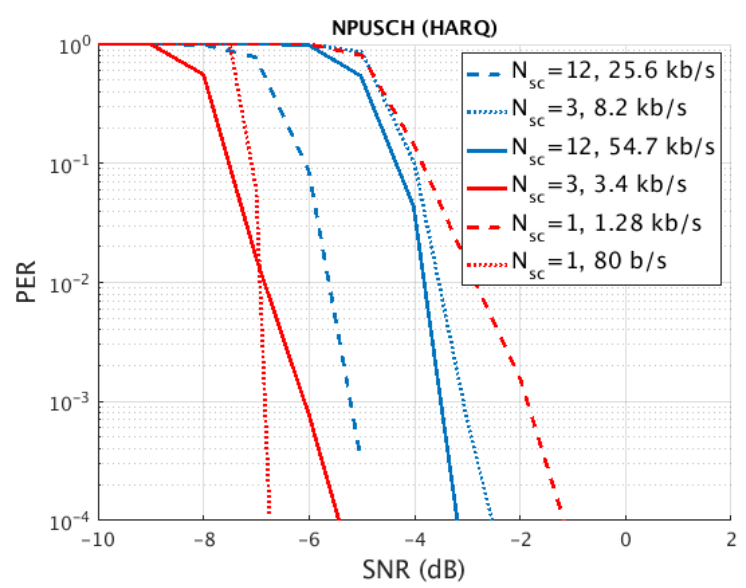

Fig. 7. NPUSCH performance.

Fig. 8 shows the NPRACH detection performance with the evolution of the non-detection rate as a function of the SNR 
for different repetition rates for the GEO scenario (no CFO) and LEO scenario with $1 \mathrm{kHz}$ of CFO (maximum tolerance in UL). This evaluation allows us to determine the number of repetitions required to detect the random access preamble for the studied scenarios. The detection of NPRACH channel is then possible without repetition for the LEO scenario while 8 repetitions are required for the GEO scenario.

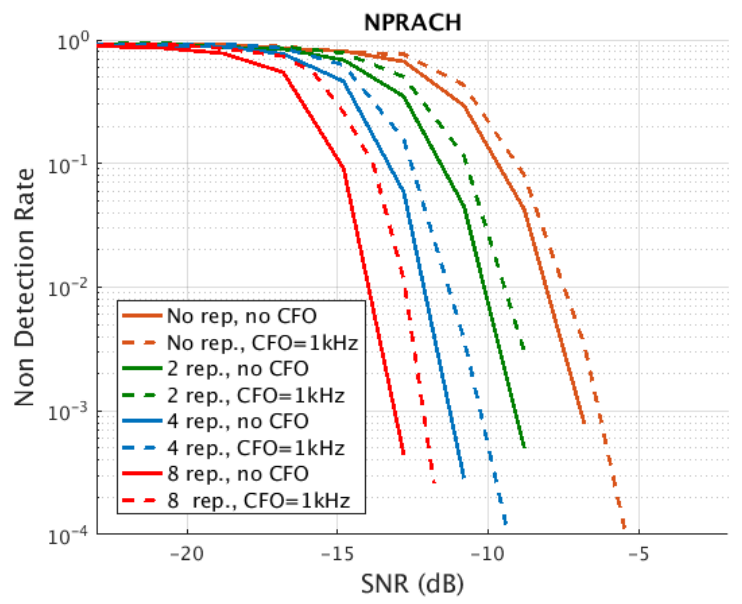

Fig. 8. NPRACH performance.

\section{CONCLUSION}

IoT services by satellite is highly recommended to provide worldwide IoT connectivity. In this paper, we demonstrated that NB-IoT could be a good candidate for this context. We analyzed the compatibility of terrestrial NB-IoT with bidirectional satellite links for GEO and LEO scenarios. Both satellite communication scenarios have been studied to evaluate key performance criterion for the link: these include Doppler levels and link budget. A new reception strategy has then been proposed in order to be able to demodulate and decode the NB-IoT channels in both UL and DL. New dedicated reception algorithms were developed and tuned for these application scenarios. To prove how compatible the NB-IoT physical layer was with the selected satellite scenarios, we evaluated the performance on the UL and DL. While Doppler levels and variations can be significantly large for the LEO scenario, the main limiting factor has been in the link budget for GEO scenario. The LEO scenario presented the best performance in terms of data rate. For the GEO scenario, performance has been affected by the distance between the satellite and the terminal. To be able to transmit at a reasonable data rate in this latter case, it is required to increase the UE transmission power. This analysis has been performed solely on physical layer performance. In a future work, we intend to include these results to analyze the impact of performance when also upper layers procedures are considered.

\section{REFERENCES}

[1] K. L. Lueth, "State of the IoT 2020: 12 billion IoT connections, surpassing non-IoT for the first time," Tech. Rep., IoT Analytics, Nov. 2020.

[2] M. Conti, A. Guidotti, C. Amatetti, and A. Vanelli-Coralli, "Nb-iot over non-terrestrial networks: Link budget analysis," in GLOBECOM 2020 - 2020 IEEE Global Communications Conference, 2020, pp. 1-6.
[3] M. Conti, S. Andrenacci, N. Maturo, S. Chatzinotas, and A. VanelliCoralli, "Doppler impact analysis for nb-iot and satellite systems integration," in ICC 2020 - 2020 IEEE International Conference on Communications (ICC), 2020, pp. 1-7.

[4] O. Kodheli, S. Andrenacci, N. Maturo, S. Chatzinotas, and F. Zimmer, "Resource allocation approach for differential doppler reduction in nb-iot over leo satellite," in 2018 9th Advanced Satellite Multimedia Systems Conference and the 15th Signal Processing for Space Communications Workshop (ASMS/SPSC), 2018, pp. 1-8.

[5] Y. E. Wang et al., "A primer on 3gpp narrowband internet of things," IEEE Communications Magazine, vol. 55, no. 3, pp. 117-123, 2017.

[6] "3rd Generation Partnership Project; Technical Specification Group Radio Access Network; Evolved Universal Terrestrial Radio Access (E-UTRA); Physical channels and modulation," Tech. Rep., Release 15, 3GPP TS 36.211 V15.2.0. June 2018.

[7] "3rd Generation Partnership Project; Technical Specification Group Radio Access Network; Evolved Universal Terrestrial Radio Access (EUTRA); Physical layer procedures," Tech. Rep., Release 15, 3GPP TS 36.213, v 15.2.0. June 2018.

[8] M. Kanj, V. Savaux, and M. Le Guen, "A tutorial on nb-iot physical layer design," IEEE Communications Surveys Tutorials, vol. 22, no. 4, pp. 2408-2446, 2020.

[9] V. Mannoni, V. Berg, F. Dehmas, and D. Noguet, "A flexible physical layer for LPWA applications," in Cognitive Radio Oriented Wireless Networks. Springer International Publishing, 2018, pp. 322-333.

[10] "LTE; Evolved Universal Terrestrial Radio Access (E-UTRA); User Equipment (UE) radio transmission and reception," Tech. Rep., (3GPP TS 36.101 version 14.3.0 Release 14), ETSI, April 2017.

[11] U. Mengali and M. Morelli, "Data-aided frequency estimation for burst digital transmission," IEEE Trans. on Communications, vol. 45, no. 1, pp. 23-25, Jan. 1997. 